\title{
g-Sign determination with circularly polarised RF fields
}

\author{
J.S. Lord ${ }^{\mathrm{a}, *}$, A. Keren ${ }^{\mathrm{b}}$, R.C. Vilão ${ }^{\mathrm{c}}$ \\ a ISIS Facility, Rutherford Appleton Laboratory, Chilton, OX110QX, UK \\ ${ }^{\mathrm{b}}$ Department of Physics, Technion-Israel Institute of Technology, Haifa 32000, Israel \\ ${ }^{\mathrm{c}}$ Physics Department, University of Coimbra, P-3004-516 Coimbra, Portugal
}

\begin{abstract}
We have used a pair of crossed coils to generate rotating RF fields for a muon spin resonance measurement by driving one of the two coils and adjusting the tuned frequencies and mutual coupling to give the required $90^{\circ}$ phase shift. Fitting the measured impedance to an equivalent circuit model gives information on the rotating field.

Test experiments on muonium in quartz confirmed the ratio of the two values of rotating field components $B_{1}$. We have then used the probe to study the shallow donor states in some II-VI semiconductors by double resonance with the electrons, where the g-factor of the electron differs from +2 and in some cases can be negative. Experiments so far have used longitudinal fields, where the double resonance can only be observed up to about $20 \mathrm{G}$ or $50 \mathrm{MHz}$.
\end{abstract}

(C) 2005 Elsevier B.V. All rights reserved.

PACS: 76.70.Dx; 76.75.+i; 71.55.Gs; 07.57.Pt

Keywords: Muon spin resonance; Semiconductor

\section{Introduction}

Most magnetic resonance experiments use linear RF magnetic fields produced by a simple coil (or singlemode microwave cavity), which is sufficient to cause transitions between spin states. In the rotating frame, on resonance $\left(\Omega=\left|\omega_{\mathrm{RF}}\right|=\gamma B_{0}\right)$ this RF field can be decomposed into two counter-rotating components, one of which causes spin flips, and the other has no effect. The direction of rotation of the reference frame depends on the sign of the gyromagnetic ratio, or g-factor for electron spin resonance.

If we can generate a rotating field, which is basically two perpendicular fields with a $90^{\circ}$ phase shift, we can determine which sense of rotation interacts with the spin, and therefore the sign of the g-factor.

\footnotetext{
*Corresponding author. Tel.: + 441235 445674; fax: + 441235445720

E-mail addresses: j.s.lord@rl.ac.uk, james.lord@rl.ac.uk (J.S. Lord).
}

\section{Experimental}

We used a double tuned RF probe, with equivalent circuit as shown in Fig. 1. This was originally designed to be used for double-resonance experiments with the two operating frequencies differing by a factor 2 or more. When the coils are tuned to nearby frequencies, the two resonances interact, appearing to "avoid" each other as the tuning capacitors are varied and one resonance is swept past the other. This interaction is due to the inevitable small mutual inductance and stray capacitance between the two coils, and we can take advantage of it.

When the two coils are tuned to the same frequency, the two resonance modes are at their closest - as seen on a network analyser connected to either probe input. Ideally we want two overlapping peaks with only a small dip between them. This can be adjusted by adding resistors in series (or alternatively in parallel) with the two tuned circuits to reduce their $Q$. The individual resonant frequency of both coils in the absence of any coupling, and our desired operating frequency, is in this dip. 


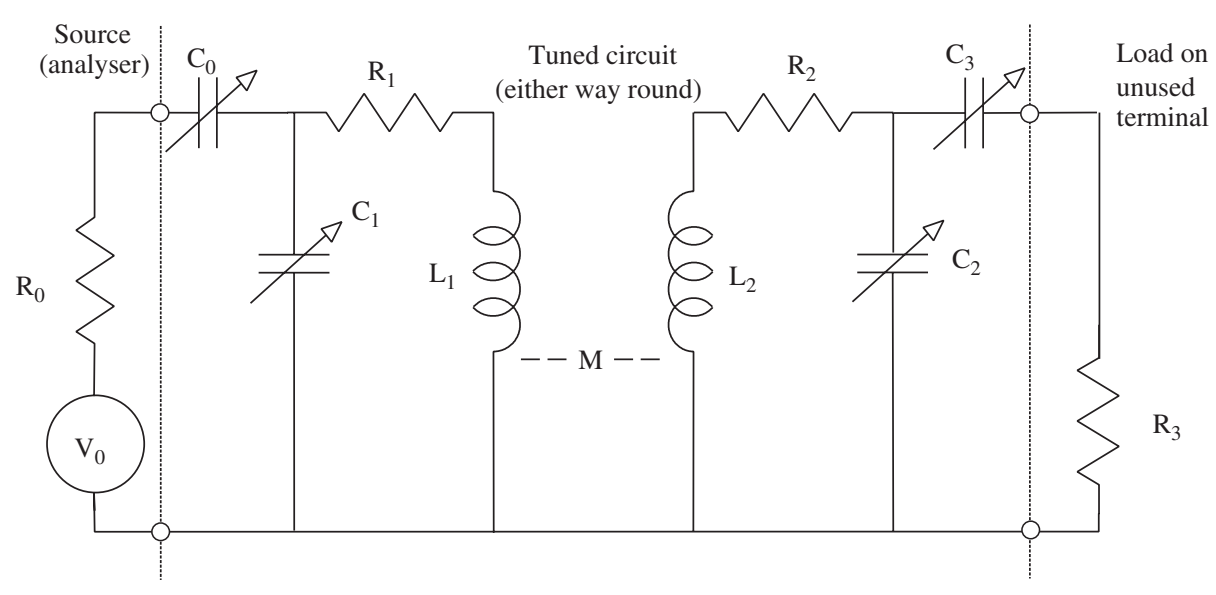

Fig. 1. The equivalent circuit of the RF probe.

We can take advantage of the coupling to generate the required phase shifts needed for a rotating field. The two coils are tuned to the same frequency, and one is driven from the RF amplifier at this frequency. The second coil, coupled only by the mutual inductance, should also resonate but with a phase shift which depends on the coupling and the $Q$ of its own resonance.

The "normal" method of driving these coils would be to use a splitter and phase shifter and two power amplifiers, one for each coil. The tuning of the coils would still be critical in order to preserve the $90^{\circ}$ phase shift.

To check the basic operation of the probe we used a small sense coil (1 turn) on a length of thin coaxial cable, and inserted it through the probe coil windings to the position of the sample. It could then be rotated about its own diameter - the axis perpendicular to the two RF $B_{1}$ fields that will be the $B_{0}$ axis in the final experiment. We monitor the phase shift between the drive RF and sense coil, and the amplitude of the sense coil signal, and find the phase changing smoothly through $360^{\circ}$ as it is rotated while the amplitude varies only slightly. Rotating field components with amplitudes in a ratio 6:1 were obtained.

Connecting the RF feed to the other coil input causes the sense of the rotation to be reversed. In this case the ratio of rotating components was only $3: 1$.

The circuit can be analysed as being composed of ideal components (L,C,R) as in Fig. 1. It is possible to express the impedance seen by the power amplifier or network analyser as a function of the component values and the frequency. In this model we consider the coupling as being due to mutual inductance but capacitance between the windings may also contribute.

$a=\frac{-j \omega M}{Z_{2}+j \omega L_{2}+R_{2}}$-ratio of currents in coils,

where

$\frac{1}{Z_{2}}=j \omega C_{2}+\frac{1}{\left(1 / j \omega C_{3}\right)+R_{3}}$
$Z=\frac{1}{\left(1 / Z_{1}\right)+j \omega C_{1}}+\frac{1}{j \omega C_{0}}-$ impedance seen by analyser,

where

$Z_{1}=R_{1}+j \omega L_{1}+j \omega M a$

$y=20 \log \frac{\left|Z-R_{0}\right|}{\left|Z+R_{0}\right|}-y_{0}$-reflection loss in $\mathrm{dB}$.

Given the equivalent components we can calculate the magnitude and phase of the current at any point, such as in the coils, as a function of frequency. In order to obtain actual fields in the coils we need to know the dimensions and number of turns. The two coils are necessarily different as one is inside the other, so the ratio of fields may be different to that of currents.

Assuming the coils are of similar size, for a rotating field the ratio of their currents $a= \pm j$ and Eq. (1) implies that $\omega^{2} C_{2} L_{2}=1$ and $\omega M=R_{2}$. The first condition means that the second tuned circuit must be on resonance, and the second requires that the coupling is matched to the loss. If the coils differ in size or number of turns, this would require $a= \pm j k$ with $k \neq 1$ and therefore $\omega M=k R_{2}$ which is just as easily satisfied by choosing $R_{2}$.

We can measure the reflection coefficient $y(\omega)$ with a network analyser and fit it to obtain the unknown values of $L$ and $C$ (the resistors $R_{1}$ and $R_{2}$ and the impedance of the source $R_{0}=50 \Omega$ have known values). Measurements are taken at both terminals for the same tuning condition and used in a simultaneous fit. Fig. 2 shows the measured and simulated responses of the ideal circuit, for one of the two inputs.

\section{Calibration}

We used the muonium "triplet" resonance in quartz to check the operation of the rotating-field coil for a real muon experiment. As a compact species, the electron in muonium will have $g=+2$ so the triplet resonance appears 
at $\omega=\frac{1}{2}\left(g \mu_{\mathrm{B}} / \hbar-\gamma_{\mu}\right) B$ or an effective gyromagnetic ratio of approximately half that of the free electron.

We used the MuSR instrument [1] in longitudinal geometry, with additional coils used to offset the longitudinal field by $25 \mathrm{G}$ enabling a smooth sweep through zero to the resonance positions at either side. The coil was tuned to $23 \mathrm{MHz}$ giving an expected resonance at $18 \mathrm{G}$. The RF pulse length was set to cover the whole period of muon implantation and decay. We measured the difference in asymmetry with the RF turned on and off to separate out the effect of the RF resonance from other low field relaxation effects.

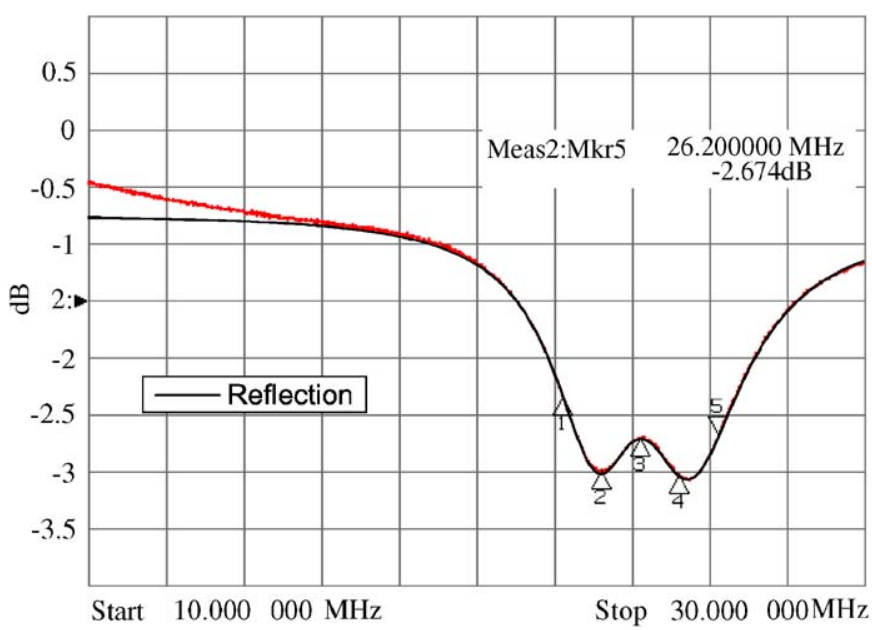

Fig. 2. The electrical resonance measured on the network analyser, and the simulated curve from the ideal circuit with fitted component values.
Fig. 3 shows the results. The line is visible for both signs of $H_{0}$ but the resonance for $H_{0}<0$ is much broader than that for $H_{0}>0$ and in addition the "wiggles" seen on resonance in the time domain (insets Fig. 3) are at higher frequency, indicating higher effective $H_{1}$.

We find fields of $H_{1}^{+}=1.27 \pm 0.04$ and $H_{1}^{-}=$ $0.45 \pm 0.05 \mathrm{G}$ for an RF power level of $-23 \mathrm{dBm}$ at the oscillator (about $12 \mathrm{~W}$ output from the power amplifier).

\section{Measurements}

The objective of this experiment was to measure the sign of the g-factors of some shallow donor states in II-VI semiconductors. The states have been identified by their transverse field spectra [2-5] and comprise an interstitial muon with a weakly bound electron, and hyperfine constant below $1 \mathrm{MHz}$. The observation of a value $g \neq+$ 2 confirms that we are not measuring a compact radical state [6]. CdTe should have $g=-1.6$ while CdS has $g=$ +1.78 and previous measurements with linear RF fields have confirmed the magnitudes of these values for muon centres. The determination of a negative g-value would be a striking demonstration of the conduction-electron nature of the shallow centre.

The method uses an RF field to cause the electron spin to flip, thus decoupling the muon from the electron. The dominant interaction on the electron is its Zeeman splitting in the applied field, so its resonant frequency is approximately $\hbar \omega=g \mu_{\mathrm{B}} B$. A sufficiently large RF amplitude causes the electron to flip more rapidly than the hyperfine coupling between it and the muon, so the muon interaction is motionally narrowed. In transverse geometry this results

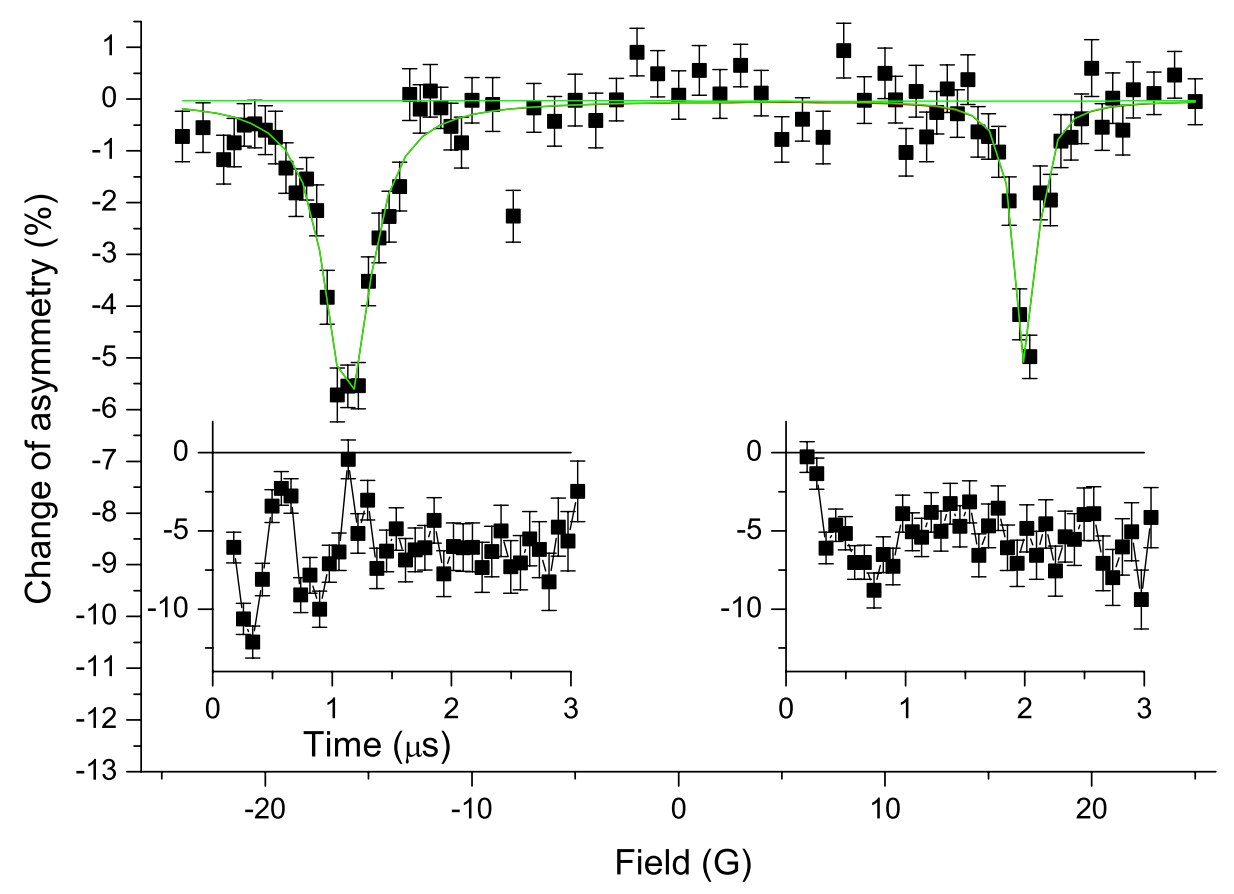

Fig. 3. Calibration measurement of the muonium triplet resonance in quartz. Insets: time domain signals on resonance. 
in the two line spectrum collapsing to a single line at the diamagnetic frequency. In longitudinal geometry the muons are no longer depolarised at the level-crossing dip, so leading to resonant repolarisation - the opposite sense to most resonances.

The shallow donor resonance was performed in longitudinal geometry, as described in [7]. In order for a signal to be observed in this mode, the longitudinal field must be fairly close to the level crossing field in order for there to be some polarisation to recover when on resonance. In addition the hyperfine coupling of the shallow donor must be sufficiently anisotropic that the level crossing is observable. With an isotropic hyperfine coupling the levels cross without interaction and no change of longitudinal polarisation can be observed.

We kept the probe tuned to $23 \mathrm{MHz}$ as for the calibration, corresponding to a resonant field of $8 \mathrm{G}$ (if $g=2$ ) or a hyperfine coupling around $220 \mathrm{kHz}$. The samples of $\mathrm{CdS}$ and $\mathrm{CdTe}$ were cooled to the base temperature of the helium cryostat (about $1.4 \mathrm{~K}$ ) to ensure the shallow donors are not ionised and have a long spin lifetime.

The amplitude of the RF field $H_{1}$ used has to be chosen so that the rotating component decouples the electron from the muon while the counter-rotating component does not. Since the ratio of rotating components of field is fixed for a given tuning condition, a sufficiently large $H_{1}$ would give resonances at both sides of zero.

We measured a single crystal plate of CdS oriented perpendicular to [100]. As it is hexagonal, the bonds and hyperfine axes necessarily make different angles to the field, which could broaden the resonances.

$\mathrm{CdS}$ is known to give a large fraction of the shallow donor state, and has a sufficiently large anisotropy of the shallow donor coupling to give a good signal in longitudinal geometry.

We measured with RF power of $-23 \mathrm{dBm}$ (as for the calibration) and also $-29 \mathrm{dBm}$ (a factor 2 lower in $H_{1}$ ). The resonance requires $\gamma_{e} H_{1}>A$ so for our samples where $A \sim 250 \mathrm{kHz}$ we need $H_{1}^{+}>0.1 \mathrm{G}$ and ideally $H_{1}^{-}<0.1 \mathrm{G}$.

The results in Fig. 4 show a large resonant "repolarisation" peak centred at zero true field, overlapping with the smaller peak near the expected resonance field of $-9 \mathrm{G}$, which is on the side corresponding to positive $g$ as expected. The nature of the zero-field resonance is as yet unknown - though it was not observed with the quartz calibration sample at room temperature.

The CdTe sample was oriented along [ $\left[\begin{array}{ll}1 & 0\end{array}\right]$ and cooled to the base temperature. The cubic structure has all the $\mathrm{Cd}-\mathrm{Te}$ bonds and probable hyperfine anisotropy axes along [ 1111 ], at $54^{\circ}$ to the applied field, a good orientation to observe the level crossing resonance.

CdTe shows a co-existence of "normal" muonium and the shallow-donor state, and the resonance line from the normal muonium was visible at the same field as for the quartz calibration sample. This is the first direct observation that this muonium fraction is present on a micro-

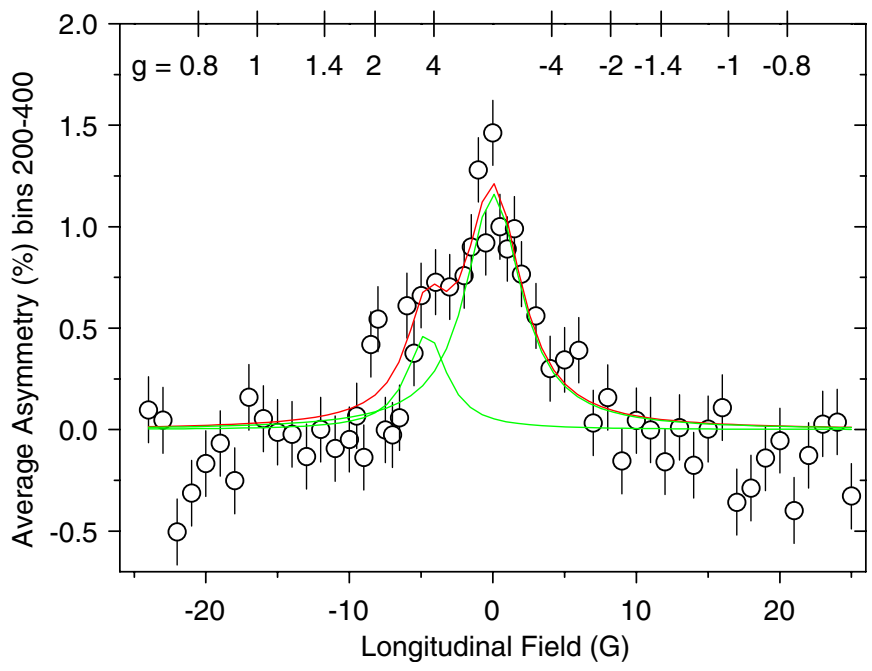

Fig. 4. Resonant repolarisation of the shallow donor state in CdS.

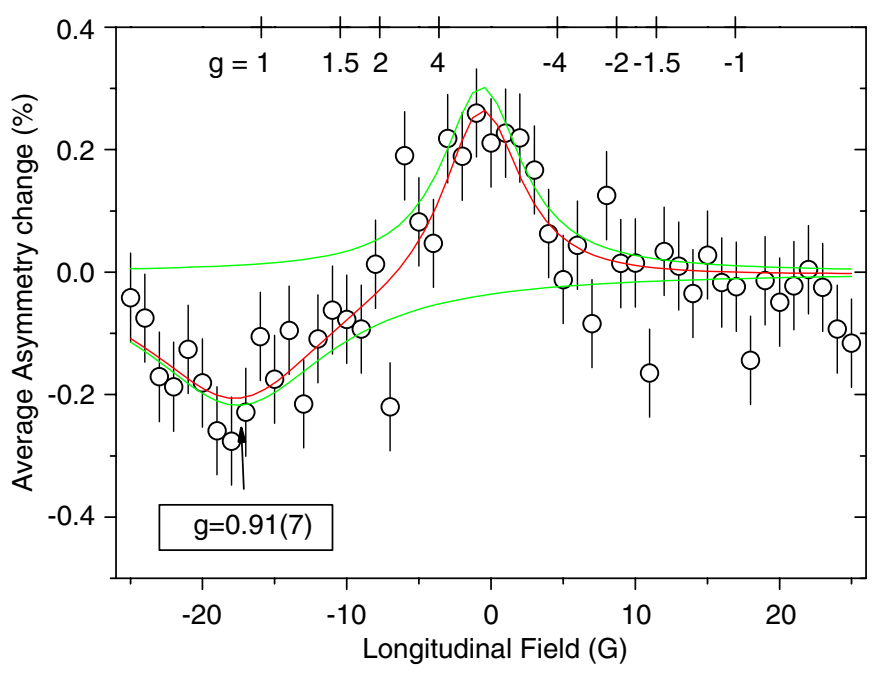

Fig. 5. Resonant repolarisation of the shallow donor state in $\mathrm{CdTe}$, also showing a resonance line from the muonium fraction.

second timescale-previous measurements have only inferred its presence from longitudinal field repolarisation.

No significant resonant signal was seen from the shallow state (Fig. 5). The large zero-field effect was present and may obscure the much smaller signal. It would be expected to be small due to the low anisotropy and low shallowdonor fraction. We only have an upper limit for the anisotropy of $40 \mathrm{kHz}$ from the transverse field spectra and it may be much smaller.

The zero field peak may be due to resonance with the electron-Cd hyperfine coupling. Further experiments at different frequencies are required.

\section{Conclusion}

We have demonstrated the feasibility of using circularly polarised RF fields for the study of muonium states. Additionally, we have undertaken the first steps in the 
corresponding research of the shallow-donor states in II-VI semiconductors. Further experiments will be needed, in transverse geometry which has a number of advantages. The field and frequency used can be higher thus separating the wanted signals from any zero field effects. The hyperfine coupling is not required to be anisotropic in order to observe the signal. Measurements in both positive and negative field directions are still required.

\section{Acknowledgements}

We are indebted to J.J. Davies, author of several of the ODMR experiments on chemical impurities and shallow dopants $[8,9]$, for suggesting g-factor measure- ments on these muonium states, as well as for invaluable discussions.

The work was performed at the ISIS Pulsed Muon facility under the auspices of EPSRC Grant GR/R53067.

\section{References}

[1] <http://www.isis.rl.ac.uk/muons/musr >

[2] J.M. Gil, et al., Phys. Rev. Lett. 83 (1999) 5294.

[3] J.M. Gil, et al., Phys. Rev. B 64 (2001) 075205.

[4] S.F.J. Cox, et al., Phys. Rev. Lett. 86 (2001) 2601.

[5] K. Shimomura, et al., Phys. Rev. Lett. 89 (2002) 255505.

[6] J.S. Lord, et al., J. Phys.: Condens. Matter 16 (2004) S4707.

[7] J.S. Lord, et al., Physica B 326 (2003) 128.

[8] P.E. Simmons, et al., Solid State Commun. 43 (1982) 311.

[9] J.L. Patel, et al., J. Phys. C Solid State 14 (1981) 1339. 